\title{
Optimisation Du Choix De La Zone Du Pompage Des Eaux De Barrage Bab Louta (Taza, Maroc)
}

\begin{abstract}
Mohamed Achmit,
Laboratoire de Chimie Analytique Appliquée, Matériaux et Environnement, Université Mohammed Premiere, BP 71760000 Oujda Maroc, BV

Mohamed VI BP 717, Oujda 60000, Maroc
\end{abstract}

\section{Ghita Sbai,}

Laboratoire des Procédés, Energies Renouvelables et Environnement, Université Sidi Mohamed Ben Abdellah, Ecole Supérieure de Technologie Route d'Imouzzer, B.P. 2427 Fès C.P. 30000, Maroc

\section{Abdelouahad Aouniti,}

Laboratoire de Chimie Analytique Appliquée, Matériaux et Environnement,

Université Mohammed Premiere, BP 71760000 Oujda Maroc, BV

Mohamed VI BP 717, Oujda 60000, Maroc

Mohammed Loukili,

Laboratoire des Procédés, Energies Renouvelables et Environnement, Université Sidi Mohamed Ben Abdellah, Ecole Supérieure de Technologie Route d'Imouzzer, B.P. 2427 Fès C.P. 30000, Maroc

doi: 10.19044/esj.2017.v13n12p231 URL:http://dx.doi.org/10.19044/esj.2017.v13n12p231

\begin{abstract}
In Morocco, the quality of water gathered in dams is deteriorating considerably due to different sources of pollution. One of the consequences of this situation is the presence of nutrients, particularly phosphorus and nitrogen, in the water. This, however, indicates an advanced state of eutrophisation of waters. This phenomenon causes an imbalance in the ecosystem with an anarchic development of seaweeds and an intense consumption of oxygen at the bottom of lakes. The present study aims to present the results of the optimization of the choice of the pumping area of the waters of the BAB LOUTA dam in TAZA region, Morocco. This dam is the dam that provides the city of TAZA with drinking water. The results of physico-chemical analyses (dissolved Oxygen, Turbidity, Manganese, $\mathrm{pH}$, and the temperature) were taken according to a vertical deep profile for a duration that spreads from March 2016 to March 2017.
\end{abstract}

Keywords: Eutrophication, dam BAB LOUTA, Oxygen dissolves 


\section{Résumé}

$\mathrm{Au}$ Maroc, plusieurs retenues de barrages connaissent des problèmes de dégradation de leur qualité d'eau due essentiellement aux différentes sources de pollution. L'une des conséquences de cette situation est l'enrichissement de l'eau en nutriments, particulièrement le phosphore et l'azote, indicateurs d'un état avancé d'eutrophisation des eaux. Ce phénomène provoque un déséquilibre de l'écosystème avec un développement anarchique d'algues et une consommation intense de l'oxygène au fond des lacs. Le présent travail consiste à présenter les résultats de l'optimisation du choix de la zone du pompage des eaux de la retenue de barrage $\mathrm{BAB}$ LOUTA région de TAZA Maroc qui sert à alimenter la ville de TAZA en eau potable. Les résultats d'analyses physicochimiques (l'Oxygène dissous, la Turbidité, le Manganèse, le $\mathrm{pH}$ et la température) ont été prelevès selon un profil profond vertical pour une durée qui s'étend de mars 2016 à mars 2017.

Mots-clés : Eutrophisation, barrage BAB LOUTA, Oxygène dissous

\section{Introduction}

$\mathrm{Au}$ Maroc, pays à climat semi-aride, le développement des agglomérations urbaines, la pression démographique et la limitation des ressources en eau souterraines font que le pays a de plus en plus recours aux eaux superficielles pour satisfaire en quantité suffisante les besoins des populations en eau potable et industrielle.

Actuellement, $65 \%$ de l'eau potable est produite à partir des ressources superficielles, principalement des retenues de barrages. Ces dernières sont souvent affectées par le phénomène d'eutrophisation qui génère un certain nombre de problèmes de qualité que le producteur d'eau potable doit affronter.

L'eutrophisation résulte d'un accroissement de la fertilisation de l'eau d'un lac par un apport d'éléments nutritifs (El Ghachtoul, Y. et al., 2005); qui favorisent la prolifération du phytoplancton et des plantes aquatiques. Peu à peu, ce processus accélère la sédimentation: le lac se rétrécit, se comble et finit par disparaître (Ramade, 2005). Ce phénomène a été classé, en 1997 par l'International Lake Environment Committee (ILEC), au quatrième rang des six risques majeurs susceptibles d'affecter les lacs et les réservoirs de la planète.

En période estivale, l'établissement de la stratification thermique, dans les lacs réservoirs, empêche le mélange des eaux de surface avec celles du fond, ce qui aggrave l'altération de la qualité de l'eau. En effet, la différence de densité des masses d'eau en liaison avec la température, crée 
une zonation dans le plan d'eau en le divisant en trois compartiments: Epilimnion, Métalimnion et Hypolimnion.

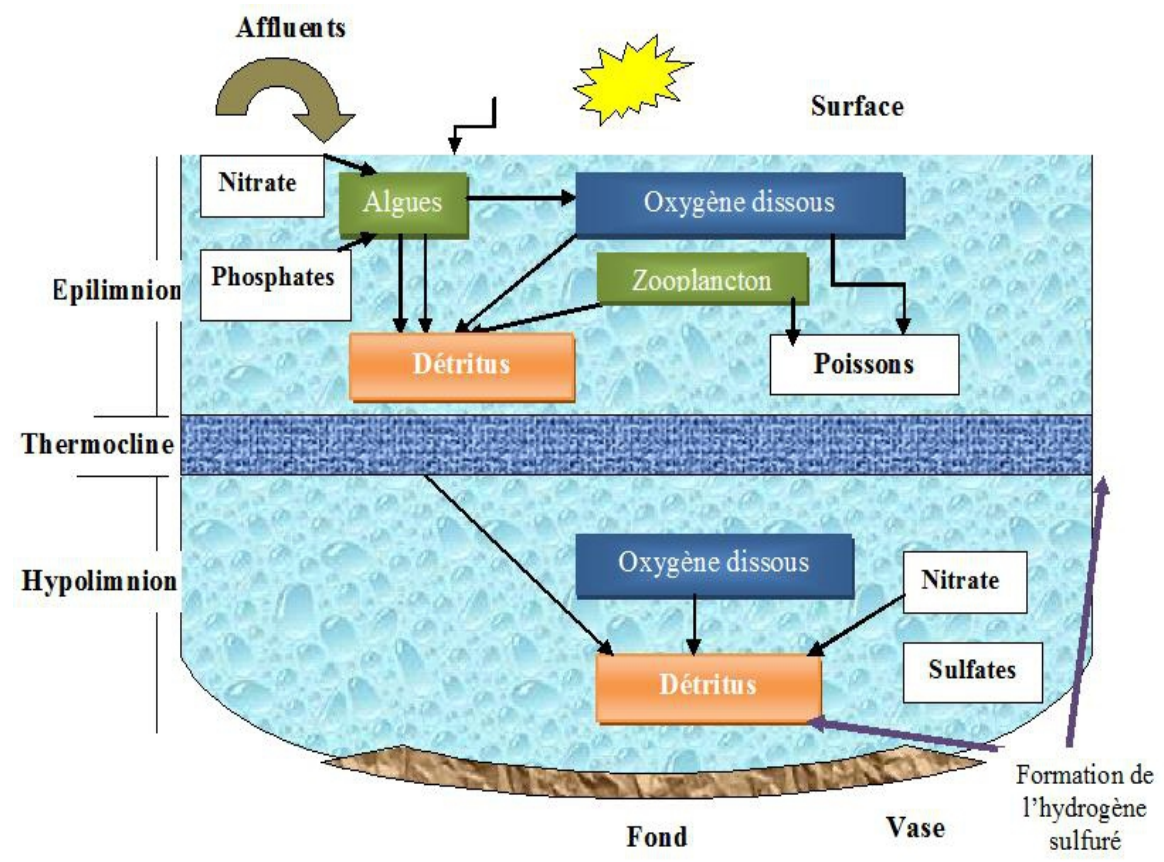

Figure 1. Répartition des zones d'eau ans un retenue de barrage

Le présent travail reflète une approche pour l'optimisation de la zone de pompoage des eaux de la retenue de barrage Bab Louta.

\section{Presentation du Site D'étude}

Le bassin versant est situé dans la province TAZA cercle TAHLA et est localisé au niveau de deux zones rurales SMIAA 5800 ha et ZERARDA 6412 ha. Alors que, une partie importante de ce bassin versant dépend du CDF de TAHLA qui relève du service provincial des eaux et forêts de Taza.

Le bassin versant de BAB LOUTA occupe une superficie de 12212 ha et s'étend entre les méridiens $4^{\circ} 23$ et $4^{\circ} 10$ ouest et le parallèle $33^{\circ} 04$ nord. Il est situé entièrement dans le moyens atlas tabulaire. L'altitude maximale atteint $1554 \mathrm{~m}$ et l'altitude minimale $530 \mathrm{~m}$. l'altitude médiane est de $926 \mathrm{~m}$ .quatre classes d'altitude sont retenus : moins de $700 \mathrm{~m}$ couvrant $12.6 \%$ entre 700 et $1100 \mathrm{~m}$ occupant $53.1 \%$, entre 1100 et $1500 \mathrm{~m}$ sur $33 \%$ et plus de $1500 \mathrm{~m}$ sur $1.3 \%$ de la superficie totale. 


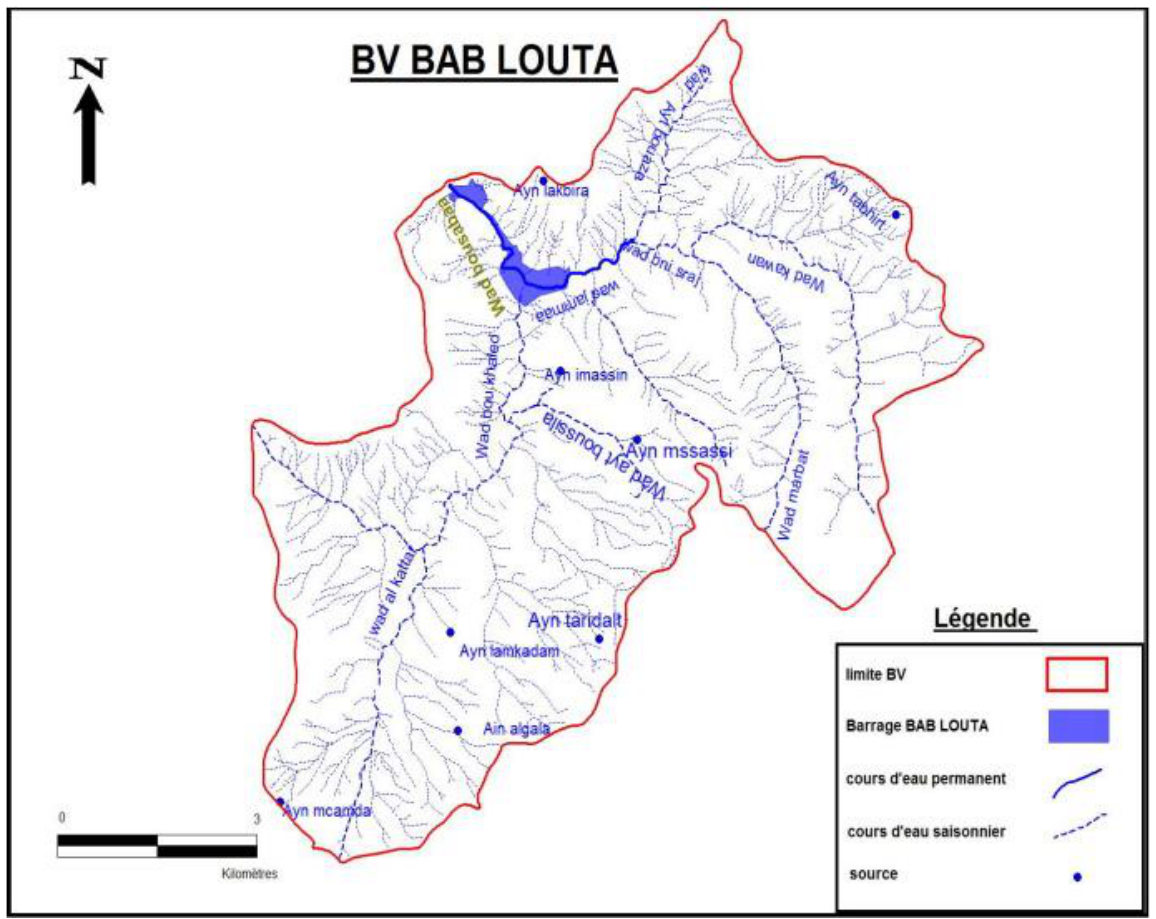

Figure 2. Carte hydrographique du Bassin versant BAB LOUTA

Le BV de BAB LOUTA est drainé OUED BOUSBAA qui constitué par 2 cours d'eau:

+ Coté Sud-Est oued JMAA.

+ Coté Est oued béni SERAJ.

D'où le $\mathrm{BV}$ est constitué de deux principaux sous-bassins versant est le plus important le sous bassin drainé par l'oued BOUKHALED il est constitué de deux principaux bassin intermédiaire qui représentent 6 et $9 \%$ de la superficie totale du bassin versant.

Les longueurs des principaux cours d'eau sont relativement important puisqu'elles avoisinent $14 \mathrm{Km}$, les pentes de cours d'eau sont fortes du fait ils peuvent atteindre $16 \%$, en effet de chaque examiné des profils des deux principes OUED montre que les pentes sont très variables fortes au niveau des tronçons amont des OUED et faible au tronçon aval, alors cette variation des pentes permet d'arrachés des matériaux le long des tronçons aval avant d'atteindre la retenue du barrage. 
La température varie entre $20.6^{\circ} \mathrm{C}$ à TAHLA et $11.6^{\circ} \mathrm{C}$ à TAZEKA .Les vents dominants dans la BV:

$>$ Les vents continentaux secs: ce sont les vents de l'EST et SUD-EST (chergui) leur action est des séchante.

Les vents océaniques humides: ce sont les vents de l'OUEST et NORD-EST qui ont un effet bénéfique sur la végétation.

Tableau 1. Caractéristiques morphométriques et hydrologiques du barrage BAB

\begin{tabular}{|l|l|}
\multicolumn{2}{l}{ LOUTA } \\
\hline Année de construction & 1999 \\
\hline Cours d'eau & Oued Bousbaa \\
\hline Centre le plus proche & Tahla \\
\hline Province & Taza \\
\hline But de l'ouvrage & $\begin{array}{l}\text { Alimentation en eau potable de la ville } \\
\text { de Taza }\end{array}$ \\
\hline Capacité de retenus & $37 \mathrm{Mm}^{3}$ \\
\hline Apport moyens annuels & $23 \mathrm{Mm}^{3}$ \\
\hline $\begin{array}{l}\text { Caractéristiques de l'ouvrage : } \\
\text { - Type }\end{array}$ & Poids en BCR \\
- Hauteur sur fondation & $55 \mathrm{~m} \mathrm{~m}^{3}$ \\
\hline Durée des travaux & $45000 \mathrm{~m}^{3}$ \\
\hline
\end{tabular}

\section{Méthodologie}

Face au problème d'eutrophisation, l'évaluation de la qualité des eaux nécessite une connaissance, aussi complète que possible, des caractéristiques physicochimiques, chimiques des eaux. A cet effet, quatre variables (Température de l'eau, $\mathrm{pH}$, turbidité, $\mathrm{O}_{2}$ dissous, Manganèse).

Les prélèvements d'eau ont été effectués trimestriellement pendant la période mars 2016 à Mars 2017 à des profondeur différents car le profondeur est un élément déterminant le fonctionnement écologique des lacs, pour cela nous avons utilisés la bouteille de Van Dorn. En suivant les méthodes standardisées (Afnor, 1997; Rodier, 1996) cinq variables ont fait l'objet d'un suivi physico-chimique.

Sur l'ensemble des échantillons prélevés, six paramètres ont été mesurés: Le $\mathrm{pH}$ et la température de l'eau à l'aide d'un $\mathrm{pH}$ mètre Type HACH-Modèle Sension 7, la turbidité a l'aide d'un turbidimètre type turbidimètre Type HACH Modèle $2100 \mathrm{~N}$ l'oxygène dissous par un Oxymètre Type HQ Modèle $40 \mathrm{~d}$ multi et le manganèse par Kit de Manganèse. 


\section{Résultats et Discussion \\ Température de L'eau}

La température de l'eau, est un facteur écologique qui entraîne d'importantes répercutions écologiques (Leynaud, 1968). Elle agit sur la densité, la viscosité, la solubilité des gaz dans l'eau et la dissociation des sels dissous. Elle a aussi un effet sur les réactions chimiques et biochimiques, sur le développement et sur la croissance des organismes vivants dans l'eau, et particulièrement les microorganismes (W.H.O.,1987).

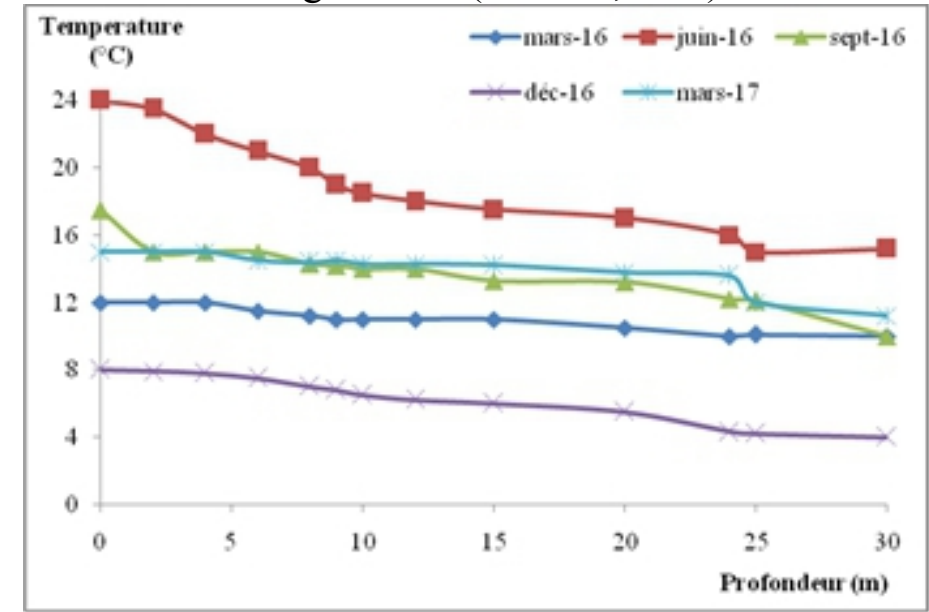

Figure 3. Variation de la temperature en differents saisons en fonction de profondeur

Du point de vue régime thermique, les eaux de la retenue de barrage BAB LOUTA se caractérisent par une stratification qui s'étale au juin 2016 (Figure 3). Globalement, les valeurs de température oscillent entre $8^{\circ} \mathrm{C}$ et $24^{\circ} \mathrm{C}$ en surface et entre $4^{\circ} \mathrm{C}$ et $15,2^{\circ} \mathrm{C}$ au fond.

\section{Potentiel d'hydrogéne}

Le $\mathrm{pH}$ de l'eau mesure la concentration des protons $\mathrm{H}^{+}$contenus dans l'eau. Il résume la stabilité de l'équilibre établi entre les différentes formes de l'acide carbonique et il est lié au système tampon développé par les carbonates et les bicarbonates (Ezzaouaq,1991; El Blidi \& Fekhaoui, 2003; Himmi et al., 2003). 


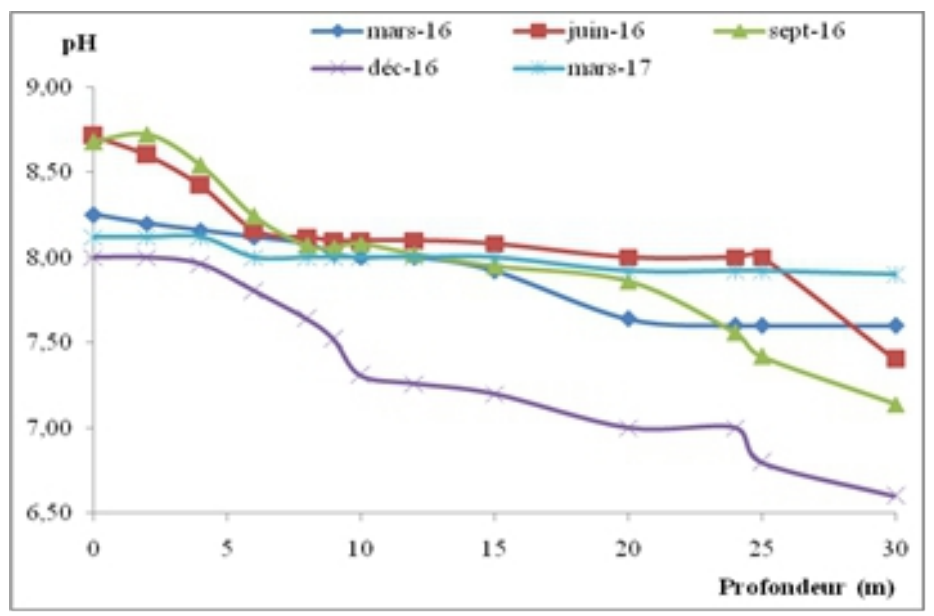

Figure 4. Variation de pH en differents saisons en fonction de profondeur

Le $\mathrm{pH}$ de l'eau demeure légèrement alcalin. Le valeurs varient globalement entre 6.60 et 8.82 (Figure 4).Les plus forte valeurs sont enregistrées en zone superficielle, en raison d'une importante activité photosynthetique.la plus forte valeur est enregistrée en septembre 2016.

\section{Turbidité}

La turbidité est une propriété qui renseigne sur la limpidité d'une eau. Elle dépend de matières colloïdales dans l'eau (limons, argiles, microorganismes...).

La turbidité est l'un des paramètres de contrôles de la qualité des eaux. La turbidité s'exprime en NTU (Unité de Turbidité Néphélométrie).

Le paramètre de la turbidité ne semble pas être de bon descripteur de la qualité d'eaux de ce retenue se trouvant dans la zone semi aride du fait de l'importance de la charge solide. La figure 5 montre que les valeurs de la turbidité aux zones épilimnion et thermocline sont négligeables par rapport aux valeurs trouvées à la zone hypolimnion c'est-à-dire à la profondeur de $25 \mathrm{~m}$ et plus. 


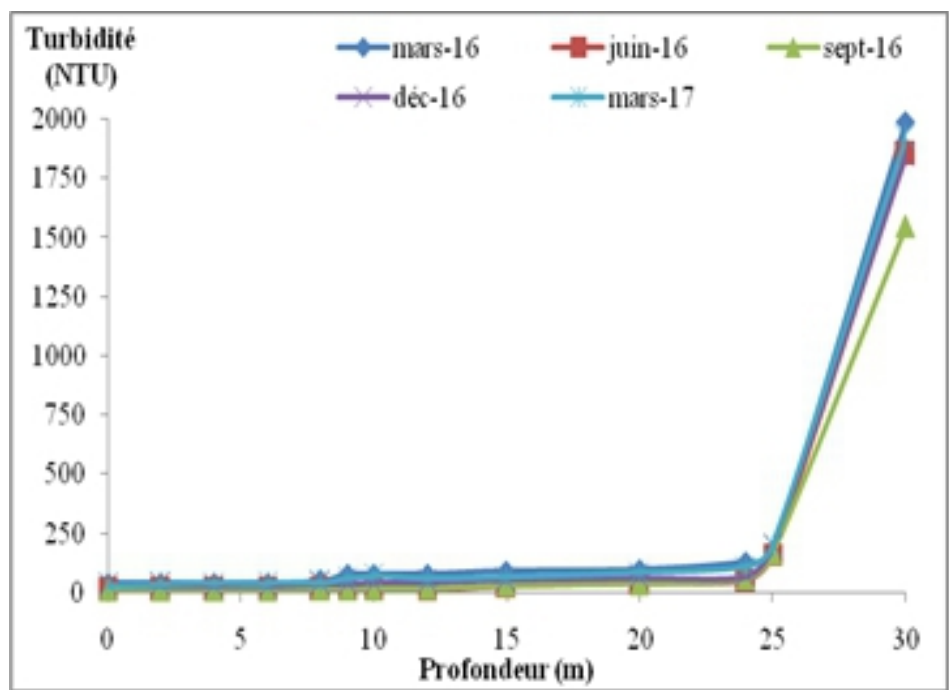

Figure 5 . Variation de la turbidité en differents saisons en fonction de profondeur

\section{Manganése}

Les concentrations en manganèse au fond oscillent entre $0.2 \mathrm{mg} / 1$ et $0.8 \mathrm{mg} / \mathrm{l}$. Les fortes valeurs sont dues au relargage du manganèse en provenance des sédiments dans les conditions anoxiques. Les plus fortes valeurs sont enregistrées en Septembre et Décembre 2016 (Figure 6).

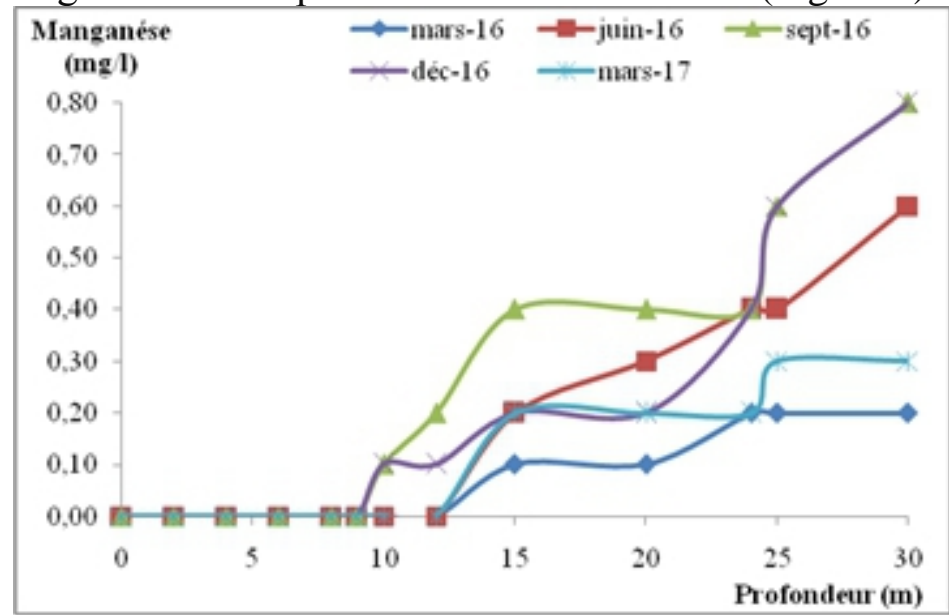

Figure 6. Variation de la concentration de Manganése en differents saisons en fonction de

\section{Oxygéne Dissous}

profondeur

La figure 7 montre que les couches superficielles de la retenue sont bien oxygénées durant la période d'étude ; les valeurs de l'oxygène dissout a la surface varie entre $4,24 \mathrm{mg} \mathrm{O}_{2} / 1$ et $9,33 \mathrm{mg} \mathrm{O}_{2} / 1 \mathrm{La}$ valeur maximale est enregistré au mois de Mars 2016 et la valeur minimal enregistré au mois de Septembre 2016. Ce paramètre diminue progressivement en fonction de 
profondeur, au profondeur de 30 mètre il prend des valeur entre $2 \mathrm{mg} \mathrm{O}_{2} / \mathrm{l}$ et $5,6 \mathrm{mg} \mathrm{O} / 2$.

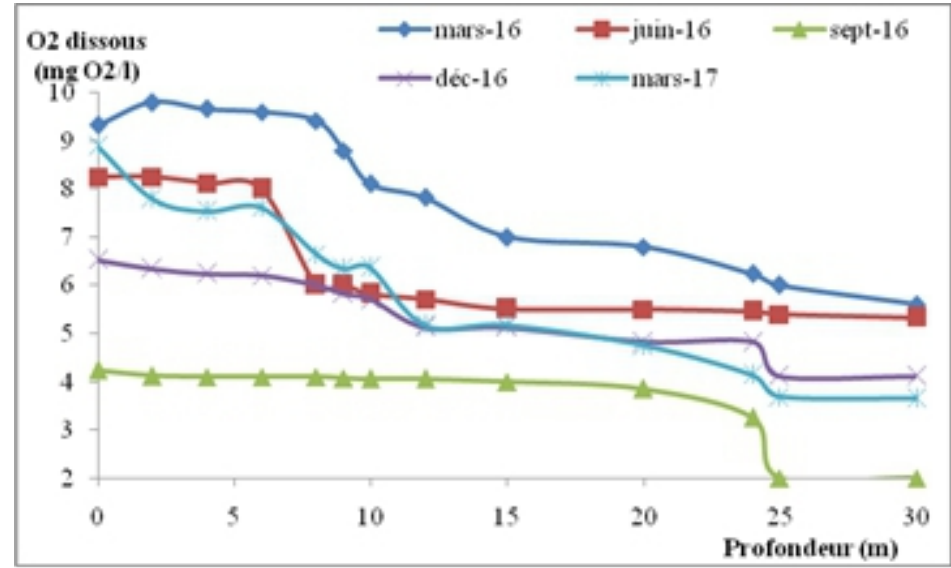

Figure 7. Variation de l'oxygéne dissous en differents saisons en fonction de profondeur

\section{Optimisation du Niveau de Prise D'eau}

Étant donné, que la qualité de l'eau dans une retenue, change dans le temps et dans l'espace, notamment en fonction de la profondeur lors de la stratification thermique, le choix du niveau de prise d'eau dans la retenue peut jouer un rôle important dans l'amélioration de la qualité de l'eau produite, et partant dans l'économie des produits de traitement utilisés, au niveau de la station de potabilisation.

Le niveau de puisement d'eau, présentant la meilleure qualité, est choisi en fonction des résultats de la stratification des paramètres de la qualité des eaux les plus importants (température, $\mathrm{pH}$, oxygène dissous, turbidité et manganèse).

A noter que l'eau brute alimentant les stations de traitements doit répondre dans la mesure du possible aux critères suivants:

- Température aux environs de $15^{\circ} \mathrm{C}$

- $\mathrm{pH}$ voisin de 7.6 unité $\mathrm{pH}$ (meilleure floculation),

- Oxygène dissous de l'ordre de quelques milligrammes par litre,

- Turbidité minimal

- Demande en chlore minimale

- Concentration en algues minimale, avec si possible, absence de filaments et de nanoplancton

- Concentration en manganèse les plus faibles possibles 
L'étude des caractéristiques physico-chimiques, chimiques et des eaux prélevées dans le barrage étudié, de BAB LOUTA a pris l'étude des résultats d'analyse de stratification relatives au prélèvement du 01/03/2017 montrent ce qui suite:

$>$ L'écart de température entre la surface et le fond de la retenue est de $3.8^{\circ} \mathrm{C}$.

$>$ Les teneures en Oxygène dissous oscillent entre $8.88 \mathrm{mg} \mathrm{d'} \mathrm{O}_{2} / 1$ dans les eaux de surface et $3.65 \mathrm{mg} \mathrm{d} \mathrm{d}_{2} / \mathrm{l}$ dans les eaux du fond de la retenue.

$>$ Les valeurs de $\mathrm{pH}$ mesurées varient entre 8.12 unités de $\mathrm{pH}$ dans les eaux de surface et 7.90 unités de $\mathrm{pH}$ au fond de la retenue.

$>$ Les valeurs de turbidité enregistrées varient entre 30.18 NTU dans les eaux de surface et 1922 NTU dans les eaux du fond de la retenue.

D'après ces résultat obtenue il reste conseillé de puiser l'eau a partir de la zone Thermocline pour avoir l'eau de qualité optimum afin d'économiser les réactifs et faciliter le traitement de potabilisation des eaux.

\section{Conclusion}

Dans un climat semi aride comme celui du Maroc, la lutte contre les conséquences de l'eutrophisation sur la qualité des eaux de retenue de barrage BAB LOUTA utilisées pour la production de l'eau nécessite des mesures curatives pour améliorer la qualité de l'eau.et proposer des solution efficace, parmi les solution efficace il y a:

$>$ Le choix du niveau de prise d'eau brute de qualité optimal et les vidange des eaux du fond permettent de faire des économies substantielles sur le cout du traitement et améliorer la qualité gustative de l'eau produite.

$>$ L'introduction dans les retenues eutrophies, des consommateurs primaires (poisson phytoplanctonophage) a été conçue comme une correction directe de l'accumulation de la biomasse phytoplanctonique qui est à l'origine de toute la gamme de phénomènes nocifs pour la qualité de l'eau des retenues de barrage. Cette solution biologique, qui consiste en l'introduction de la carpe argentée de Chine.

$>$ Déstratification artificielle des eaux par l'installation du système d'aération, par injection d'air comprimé à travers des tubes perforés déposés près du fond du lac. La diffusion d'air dans ce cas se fait verticalement. 


\section{References:}

1. Afnor (1997). Qualité de l'eau. Recueil des normes françaises environnement. Tomes 1, 2, 3 et 4. $1372 \mathrm{p}$

2. El Blidi S. \& Fekhaoui M. (2003). Hydrologie et dynamique marégraphique de l'estuaire du Sebou (Gharb, Maroc). Bulletin de l'Institut Scientifique, Rabat, $25: 57-65$.

3. El Ghachtoul, Y. et al. (2005). Eutrophisation des eaux des retenues des barrages Smir et Sehli (Maroc): Causes, conséquence et consignes de gestion. Revue des Sciences de l'Eau, 75-89.

4. Ezzaouaq M. (1991). Caractérisation hydrodynamique, physicochimique et bactériologique des eaux superficielles de l'estuaire du Bouregreg (Maroc) soumis aux rejets des villes de Rabat-Salé. Thèse D.E.S. fac. Sci. Rabat, 140p.

5. Himmi N., Fekhaoui M., Foutlane A., Bourchic H., El Maroufy M., Benazzout T., \& Hasnaoui M. (2003). Relazione plankton-parametri fisici chimici in un bacino dimaturazione (laguna mista beni slimane - Morocco. Rivesta di idrobiologia. Universitadegli studi di Perugia, departemento di biologia animale ed ecologia laboratoire di idrobiologia "G.B. Grassi", 110-111p.

6. Leynaud G. (1968). Les pollutions thermiques, influence de la température sur la vie aquatique. B.T.I. Ministère de l'agriculture, 224-881.

7. Ramade, F. (2005). Elément d'écologie: Ecologie appliquée. Action de l'homme sur la biosphère. Paris, France: McGraw-Hill.

8. Rodier J. (1996). L'analyse de l'eau naturelle, eaux résiduaires, eau de mer, 8ème édition, Dunod, Paris, 1383 p.

9. W.H.O. (1987). Global pollution and health results of related environmental monitoring. Global environment monitoring system, WHO, UNEP. 